\title{
Endoscopic Lung Volume Reduction An American Perspective
}

Hans J. Lee*, Samira Shojaee*, and Daniel H. Sterman

Ann Am Thorac Soc Vol 10, No 6, pp 667-679, Dec 2013

\section{Background:}

Severe emphysema is an important problem, affecting $\sim 10 \%$ of people in the USA and is the fourth leading cause of death ${ }^{1}$. Treatment options include smoking cessation, inhaled medications, systemic steroids, pulmonary rehabilitation, supplemental oxygen and lung transplantation. Lung volume reduction surgery (LVRS) "reduction pneumoplasty" was first described in 1957 by Otto Brantigan ${ }^{2}$ and was later reintroduced and refined by Joel Cooper ${ }^{3}$. In the 1990s the National Institute of Health (NIH) launched a trial of medical therapy vs. surgical treatment using lung volume reduction to see if these patients would have an alternative strategy to treat this progressive disease. The goal of lung volume reduction is to remove emphysematous lung to improve hyperinflation, diaphragmatic mobility and expiratory flow. This trial was named the National Emphysema Treatment Trial (NETT) and results were published in 2003 which showed that a subset of patients who had upper lobe predominant emphysema and low baseline exercise capacity did benefit from LVRS and others did not ${ }^{4}$. A further study following patients undergoing LVRS noted a significant 90-day mortality rate in experienced hands (4\%) and post-procedural morbidity; $46 \%$ with persistent air leak requiring intercostal drainage for $>7$ days, $11 \%$ of patients with pneumonia, $7 \%$ of patients requiring intubation and $6 \%$ requiring further surgery ${ }^{5}$. Based on these results, there has been interest in development of a minimally-invasive way to reduce lung volume which can minimize morbidity and mortality. Several bronchoscopic modalities are being tested and considered for FDA approval. This article reviews all of these bronchoscopic lung volume reduction options.

\section{Methods:}

Design: Systematic review

Included Studies: Human studies, Prospective trials

Excluded Studies: Retrospective studies, Studies with $<20$ subjects, early reports if the subjects were later used in a larger cohort/trial, Non-English publications

Search terms used: "bronchoscopic lung volume reduction" or "bronchoscopy emphysema treatment" or "bronchoscopy valves" or "bronchoscopy coils" or "bronchoscopy glue" or "bronchoscopy vapor."

Databases searched: Cochrane Review, MEDLINE

Time period: Inception of database to September 2012

\section{Results:}

Valves:

The most studied of all endobronchial lung volume reduction techniques

- Reduce airflow into treated lobe during inhalation but allow air and secretions to be expelled during exhalation

- Patients who develop radiographic atelectasis show greatest benefits, those without have variable improvements.

- Improves: $\mathrm{FEV}_{1}$, lung volumes, 6 minute walk distance, health-related quality of life, St. George's Respiratory Questionnaire (SGRQ) 
- Advantage: easily reversible

- Use: heterogeneous, upper-lobe predominant emphysema without collateral ventilation

- 2 types:

o Spiration Intrabronchial valve: Umbrella-shaped device with a nitinol frame consisting 5 distal anchors and 5 proximal struts with a central rod covered in a thin layer of polyurethane.

o Zephyr valve: Duck-bill shaped, silicone-covered one way valve within a self-expanding stent that contains a flexible retainer which expands to anchor it to the airway and ensure an airtight seal.

\section{Biologic Lung volume reduction system:}

- Fibrinogen suspension and thrombin solution, which polymerize to a hydrogel as they come in contact with one another

- Induces a localized inflammatory reaction that causes atelectasis by occlusion and remodeling over 4- to 6-weeks.

- Improves FEV $1, F V C, R V / T L C, R V$, dyspnea scores and SGRQ

- Can be used in homogeneous disease, is not affected by collateral ventilation

- Irreversible

\section{AeriSeal:}

- A synthetic version of biologic lung volume reduction system

- No human blood products are used which decreases risk of transmissible diseases

- Initially uses a primer to remove surfactant which causes atelectasis and then the synthetic hydrogel is introduced which causes inflammation resulting in scarring and remodeling over several weeks

- Improves FEV $1, \mathrm{RV}$ and SGRQ

- Has been studied in upper lobe predominant disease

- Is not affected by collateral ventilation

- Irreversible

\section{Bronchoscopic thermal vapor ablation:}

- Use of heated water to produce thermal injury creating a localized inflammatory response followed by permanent fibrosis and atelectasis.

- Studied in heterogeneous, upper-lobe predominant disease

- Improves FEV $1, \mathrm{RV}, 6$-minute walk distance, BODE index, SGRQ

- Not affected by collateral ventilation

- Irreversible

\section{Coils:}

- Catheter loaded straightened nitinol coil over a guidewire. On coil deployment the straightened coil then conforms to its predetermined shape

- Coil deployment bends the airway resulting in compression of adjacent lung tissue

- Creates local lung volume reduction and restores elastic recoil of the healthier lung compartments.

- Improves SGRQ, FEV $1, \mathrm{RV}$ and 6 minute walk distance

- Not affected by collateral ventilation

- Unclear if it can be reversed

Airway bypass:

- Initially created to treat homogeneous disease 
- Placement of drug-eluting stent-supported fenestrations in the airway wall to allow for decrease in air trapping and an increased volume of air expelled during forced exhalations

- Was not successful: improvements were transient, the procedure is technically difficult and the stents are easily displaced or obstructed

\section{Commentary:}

This is a thorough review outlining all of the prospective data for the various endoscopic lung volume reduction strategies (ELVR), all in one place which makes it easy to compare one methodology to another. None of these modalities are available in the USA as they have not yet been approved by the Food and Drug Administration (FDA) but clinical trials are underway Currently, intrabronchial valves are approved only for humanitarian device exemption for persistent air leaks following segmentectomy, lobectomy and lung volume reduction surgery.

Interestingly, in the same publication of Annals of the ATS, the counterpart article from the European perspective was also published. In Europe, all of the above techniques for ELVR have been approved and are in use. Hopefully, we will have more data obtained from a larger cohort that is a more generalizable population of patients with severe COPD demonstrating benefit ${ }^{6}$.

ELVR strategies include devices that completely occlude an airway causing lobar collapse, those that mechanically decrease the lung volume and mechanisms that induce inflammation and cause subsequent fibrosis thereby decreasing lung volume. Initially, complete bilateral upper lobe treatment was attempted, but this led to pneumothoraces. Additionally, some patients were noted to have benefit without lobar collapse. This effect was postulated to be secondary to improvements in dynamic hyperinflation, collateral ventilation, ventilation/perfusion matching and redirection of ventilation to more functional alveolar units. The variables used to predict which form of bronchoscopic treatment would be most beneficial include collateral ventilation and disease heterogeneity. These characteristics are important as data suggest that indiscriminate use of ELVR strategies for patients with severe emphysema leads to clinically insignificant benefits ${ }^{7}$. In order to appropriately choose which modality will work best, one must consider and understand the concepts of collateral ventilation and disease heterogeneity.

Collateral ventilation was first described by Van Allen et al. in 1931 and is defined as alveolar ventilation via channels that bypass the usual airways. This phenomenon is negligible in normal lung but is present in two thirds of patients with severe emphysema. Physiologically, the resistance to air flow in the bronchioles is increased by mucous in patients with COPD. Increased airway resistance is then compounded by exaggerated expiratory collapse which eventually exceeds the resistance in the collateral airways thereby making them functional ${ }^{8}$.

The importance of collateral ventilation was noted during the first randomized control trial comparing medical therapy to endobronchial valve placement called the Endobronchial Valve for Emphysema Palliation Trial (VENT) ${ }^{9}$. An observation made during subgroup analysis of the European cohort of the VENT trial (Euro-VENT) ${ }^{10}$ was that the presence of a complete interlobar fissure on CT scan was an independent predictor of treatment response. When there is a defect in the interlobar fissure, ventilation takes place through the pores of Kohn which are typically only used for movement of respiratory cells such as macrophages and surfactant. Collateral ventilation is thought to derive more commonly from channels of Lambert which represent epithelium-lined tubular communications between distal bronchioles and adjacent alveoli as well as the pathways of Martin which are accessory communications between terminal bronchioles from adjacent lung segments allowing for lobar ventilation despite airway occlusion. These airflow connections therefore can occur in both interlobular as well as interlobar areas ${ }^{8}$.

In order to evaluate a patient for collateral ventilation, high resolution CT scan (HRCT) analysis of the "completeness" of the interlobar fissures has become a surrogate measure. A cutoff of $90 \%$ completeness in 1 plane on high resolution CT is used to describe a complete fissure. Anything less is considered incomplete. In a study by Aziz et al. 622 subjects with either no lung disease, or mild lung disease thought not to involve the fissures were enrolled and interlobar fissure completeness was precisely analyzed using $\mathrm{HRCT}^{11}$. As a follow-up to this information, another study of 96 patients with severe emphysema had a CT scan and an automated method was applied to quantify fissure 
completeness. Based on this analysis, emphysema was shown not to significantly affect fissure completeness by CT scan $^{12}$.

To evaluate the consistency of evaluation of high resolution CT scan for fissural integrity, CT scans of 35 patients were retrospectively reviewed by 2 pulmonologists, 1 general radiologist and 2 experienced chest radiologists, independently and blinded for treatment outcome. The pulmonary fissures were classified as either complete or incomplete. Inter-observer agreement was then assessed. They found that pulmonologists and radiologists agreed fairly well in fissure analysis, while the experienced chest radiologists reached the highest clinically adequate agreement ${ }^{13}$.

Given these challenges, a more objective, catheter-based measurement was developed to assess collateral ventilation as a reliable predictor of endobronchial valve success or failure. The system is called Chartis $^{\mathrm{TM}}$. The Chartis ${ }^{\mathrm{TM}}$ system consists of two compartments: a catheter and a console. Measurements are performed during bronchoscopy using a catheter placed in the airway of the target lung lobe. A balloon situated at the tip of the catheter allows for isolation of an area of lung. Air flow and pressure are measured and resistance of collateral channels can be calculated. A gradual decline in expiratory flow after balloon occlusion suggests that there is an absence of collateral ventilation whereas the persistence of flow after $5 \mathrm{~min}$ of balloon inflation suggests significant collateral ventilation.

The value of the Chartis ${ }^{\top M}$ system has been studied prospectively in a multicenter trial in Europe ${ }^{7,13,14,15}$ Patients were classified into two groups according to the presence or absence of collateral ventilation in the lobe of interest as determined by the Chartis ${ }^{\text {TM }}$ system and then an endobronchial valve was placed. There was a much greater volume reduction and mean percentage increase in $\mathrm{FEV}_{1}$ in the group without collateral ventilation as opposed to those found to have collateral ventilation. In a subgroup from this case series the patients were also evaluated for integrity of the lobar fissure. The accuracy in predicting a responder was similar irrespective of the method used to classify collateral ventilation status. Of note, the Chartis $^{\text {TM }}$ system is sometimes technically difficult because of low patient tolerance (coughing), incorrect positioning of the catheter secondary to difficult anatomy, or the presence of mucous which can block the catheter $^{14}$. The Chartis ${ }^{\mathrm{TM}}$ system is currently approved for clinical use in Europe but is not yet available in the United States other than for research purposes.

It has been suggested that collateral ventilation occurs to a greater extent in homogeneous emphysema than in heterogeneous disease which can explain why patients with upper lobe predominant disease fare better with ELVR in general ${ }^{16}$. Additionally, a high heterogeneity index $(\mathrm{HI})$ was predictive of successful lung volume reduction.

The $\mathrm{HI}$ is measured as the ratio of destruction between the target area for bronchoscopic lung volume reduction and another ipsilateral lobe. Patients with a higher $\mathrm{HI}$ show greater benefit with lung volume reduction than do patients with a lower $\mathrm{HI}$. A cutoff value of $15 \%$ is arbitrarily used to describe patients with high heterogeneity. The $\mathrm{HI}$ is important as patients with an elevated $\mathrm{HI}$ have evidence of lessdiseased lung on the ipsilateral side which will benefit from improved respiratory dynamics following atelectasis of the more damaged areas of lung. Despite this inherently intuitive explanation, we still have much to learn about the effects of homogeneous vs. heterogenous disease as studies on collateral ventilation have shown that patients without collateral ventilation still benefit significantly from lobar collapse despite having homogeneous disease. Therefore, patients with a low heterogeneity index should not unilaterally be excluded from lung volume reduction. HI can be determined either by interpretation of the CT scan of the chest by an experienced clinician, though this has been shown to have high inter-rater variability and therefore low reliability. Software is available now that offers more standardization and therefore more reliability to the results.

Finally, ELVR should only be used in patients with severe emphysema (FEV $120-45 \%$ and $R V>150 \%)$. The diagram below is an algorithm for bronchoscopic lung volume reduction in patients with severe emphysema.(Taken from Shah PL. and Herth FJF. Current status of bronchoscopic lung volume reduction with endobronchial valves. Thorax 2014;69:280-286) ${ }^{7}$ 


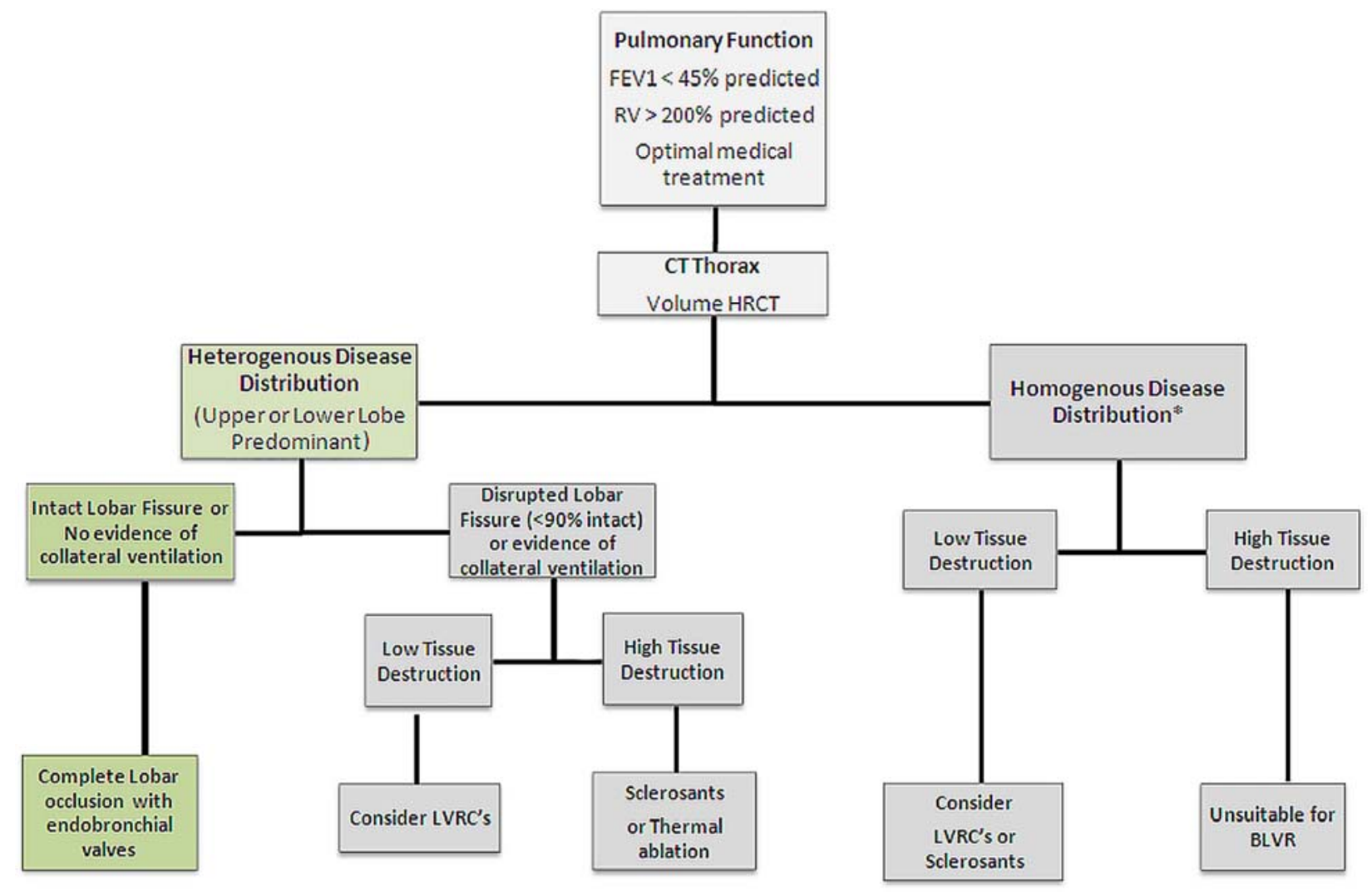

This paper's limitations include the exclusion of retrospective studies and reports of ELVR with less than 20 patients which may still provide valuable information.

Overall, this paper is an extremely helpful overview of ELVR for the Interventional Pulmonologist. ELVR appears to be safe and will hopefully become an attractive minimally-invasive alternative for patients with severe COPD. Although, ELVR remains experimental, emerging data is promising. Refining patient selection and measurements to best qualify and quantify improvement outcomes is the current challenge.

\section{Authors}

Christine Argento, MD, FCCP

Assistant Professor of Medicine Interventional Pulmonology

Emory University

Matthew Kinsey M.D., M.P.H.

Assistant Professor

Division of Pulmonary and Critical Care

University of Vermont College of Medicine

Momen M. Wahidi, MD, MBA

Director, Interventional Pulmonology and Bronchoscopy

Associate Professor of Medicine

Duke University Medical Center 


\section{References:}

1. Halbert RJ, Natoli JL, Gano A, Badamgarav E, Buist AS, Mannino DM.Global burden of COPD: systematic review and meta-analysis. Eur Respir J 2006;28:523-532

2. Brantigan OC. Surgical treatment of pulmonary emphysema. Md State Med J 1957;6:409-414.

3. Cooper JD, Patterson GA, Sundaresan RS, Trulock EP, Yusen RD, Pohl MS, Lefrak SS. Results of 150 consecutive bilateral lung volume reduction procedures in patients with severe emphysema. J Thorac Cardiovasc Surg 1996;112:1319-1329, discussion 1329-1330.

4. Fishman A, Martinez F, Naunheim K, Piantadosi S, Wise R, Ries A, Weinmann G, Wood DE; National Emphysema Treatment Trial Research Group. A randomized trial comparing lungvolume reduction surgery with medical therapy for severe emphysema. $N$ Engl J Med 2003;348:2059-2073

5. Yusen RD, Lefrak SS, Gierada DS, et al. A prospective evaluation of lung volume reduction surgery in 200 consecutive patients. Chest 2003;123:1026-37.

6. Gompelmann, D., Eberhardt, R., and Herth, FJF. Endoscopic Lung Volume Reduction A European Perspective Ann Am Thorac Soc Vol 10, No 6, pp 657-666, Dec 2013

7. Shah PL, and Herth, FJF. Current status of bronchoscopic lung volume reduction with endobronchial valves Thorax 2014;69:280-286

8. Gompelmann, D., Eberhardt, R., and Herth, FJF. Collateral Ventilation, Respiration 2013;85:515520.

9. Sciurba FC, Ernst A, Herth FJF, Strange C, Criner GJ, Marquette CH, Kovitz KL, Chiacchierini RP, Goldin J, McLennan G; VENT Study Research Group. A randomized study of endobronchial valves for advanced emphysema. N Engl J Med 2010;363:1233-1244.

10. Herth FJ, Noppen M, Valipour A, Leroy S, Vergnon JM, Ficker JH, Egan JJ, Gasparini S, Agusti C, Holmes-Higgin D, et al.; International VENT Study Group. Efficacy predictors of lung volume reduction with Zephyr valves in a European cohort. Eur Respir J 2012;39:1334-1342.

11. Aziz A, Ashizawa K, Nagaoki K, Hayashi K: High resolution CT anatomy of the pulmonary fissures. J Thorac Imaging 2004; 19: 186-191.

12. van Rikxoort EM, Goldin JG, Galperin-Aizenberg M, et al. A method for the automatic quantification of the completeness of pulmonary fissures: evaluation in a database of subjects with severe emphysema. Eur Radiol 2012; 22: 302-309.

13. M. Koenigkam-Santos et al. Incomplete fissures in severe emphysematous patients evaluated with MDCT: incidence and interobserver agreement among radiologists and pneumologists. European Journal of Radiology 81 (2012) 4161- 4166

14. Gompelmann D, Eberhardt R, Michaud G, et al. Predicting atelectasis by assessment of collateral ventilation prior to endobronchial lung volume reduction: a feasibility study. Respiration 2010;80:419-25

15. Herth FJ, Eberhardt R, Gompelmann D, et al. Radiological and clinical outcomes of using Chartis $^{\circledR}$ to plan endobronchial valve treatment. Eur Respir J 2013;41:302-8

16. Higuchi T, Reed A, Oto T, Holsworth L, Ellis S, Bailey MJ, Williams TJ, Snell GI: Relation of interlobar collaterals to radiological heterogeneity in severe emphysema. Thorax 2006; 61: 409 413. 